\title{
Phase Plate Enhanced Contrast Reveals Structure of Follicular Melanin Granules
}

\author{
G. A. Camacho-Bragado*, H. Qian**, F. M. Dixon*, H. Bryant* and M. Malac** \\ * L'Oréal Recherche, Institute for Ethnic Hair and Skin Research, Chicago, IL \\ **National Institute for Nanotechnology, Edmonton, AB Canada
}

Melanin is a family of macromolecular pigments found in humans, animals and plants. In humans, melanin is responsible for hair, eye and skin color but it is also present in the inner ear and the brain stem [1]. In the skin, eye and hair, this pigment acts as a photoprotectant absorbing impinging UV radiation and dissipating it as heat. The protective action of melanin limits UV-induced DNA damage in skin [2] as well as lipid oxidation and disulphide bond cleavage that affect the mechanical properties of hair [3]. Two types of chemically distinct melanin are produced in mammals, the blackbrown eumelanin and the red-yellow pheomelanin. Eumelanin is formed by 5, 6-dihydroxyindole (DHI) and 5, 6-dihydroxyindole-2-carboxylic acid (DHICA) [4] while pheomelanin is a polymer containing 1, 4-benzothiazine units [5]. Pro-oxidative pheomelanin and anti-oxidant eumelanin are both present in hair and skin in macromolecular aggregates. Their different proportions result in a range of colors found in human hair [3,4 and refs within]. Color and other optical properties of melanin depend on its supramolecular architecture which has not been unambiguously established. Some researchers have proposed a path for mixed melanogenesis that results in a core-shell assembly of pheo and eumelanin [6,7] while others have introduced a chemical disorder model [1].

Imaging unstained specimens is often preferred to staining as it preserves features that are hindered by heavy metal stains. Follicular melanin is also a medium for excretion of heavy metals out of the body since it selectively binds to the pigment. Thus, when heavy metal staining is used, the information regarding systemic exposure to potentially toxic levels of heavy metals is lost. Moreover, recent developments in contrast enhancement techniques beyond defocus phase contrast have shown outstanding results in unstained biological specimens $[8,9]$. We have used hole-free phase plate (HFPP) imaging [8] to image internal structure of melanin grains in hair cross sections.

A study of unstained follicular melanin granules in black, red and blond hair using a thin film HFPP TEM was carried out. The contrast increase of HFPP imaging as compared to standard bright field imaging allowed us to obtain images as shown in Fig. 1 and 2 at room temperature. Although the HFPP is not a qualitative method, i.e. it cannot be used to quantitatively interpret contrast differences over various areas of a sample; it revealed internal structure of melanin grains and lateral dimensions of the internal structures, as shown by the power spectrum shown in Fig. 1. We observed a periodic array within the granules (Fig. 1) as well as a c ore-shell supramolecular array (Fig. 2) visible only when the phase plate is inserted. Energy filtered images obtained at 15 to $20 \mathrm{eV}$ also showed stronger contrast in the region between the core and the shell, as shown in Fig. 2, while the EELS mass thickness map did not show thickness variation across the melanin granule. This suggests that the energy loss in the $15-20 \mathrm{eV}$ energy range might be a surface plasma between two organic materials of different permittivity but similar mass density. Compared with HFPP imaging, the EFTEM imaging had a lower signal/noise ratio due to some practical limitations. While the HFPP imaging appears to reproduce internal structure, which suggests that HFPP imaging is more sensitive to local sample scattering properties than EELS. 


\section{References}

[1] M. L. Tran et al., Biophysical J,. 90 (2006) 743

[2] P. Meredith and T. Sarna, Pigment Cell Res., 19 (2006) 572

[3] W. S Lee. In Aging Hair, Springer-Verlag Berlin Heidelberg, 2010.

[4] J. P Ortonne and G. Prota, J. Invest. Dermatol., 101 (Suppl. 1) (1993), 82S

[5] G. Prota. Med. Res. Rev., 8 (1988) 122

[6] J. D Simon et al., Pigment Cell Melanoma Res., 22 (2009) 563

[7] G. Agrup et al., Arch Dermatol Res., 272 (1982) 103

[8] M. Malac, M. Kawasaki, M. Beleggia, P. Li and R. Egerton, Micr. \& Microanal. 16 (2010) 526

[9] K. Nagayama et. al., Microscopy Today, 18 (2010) 10
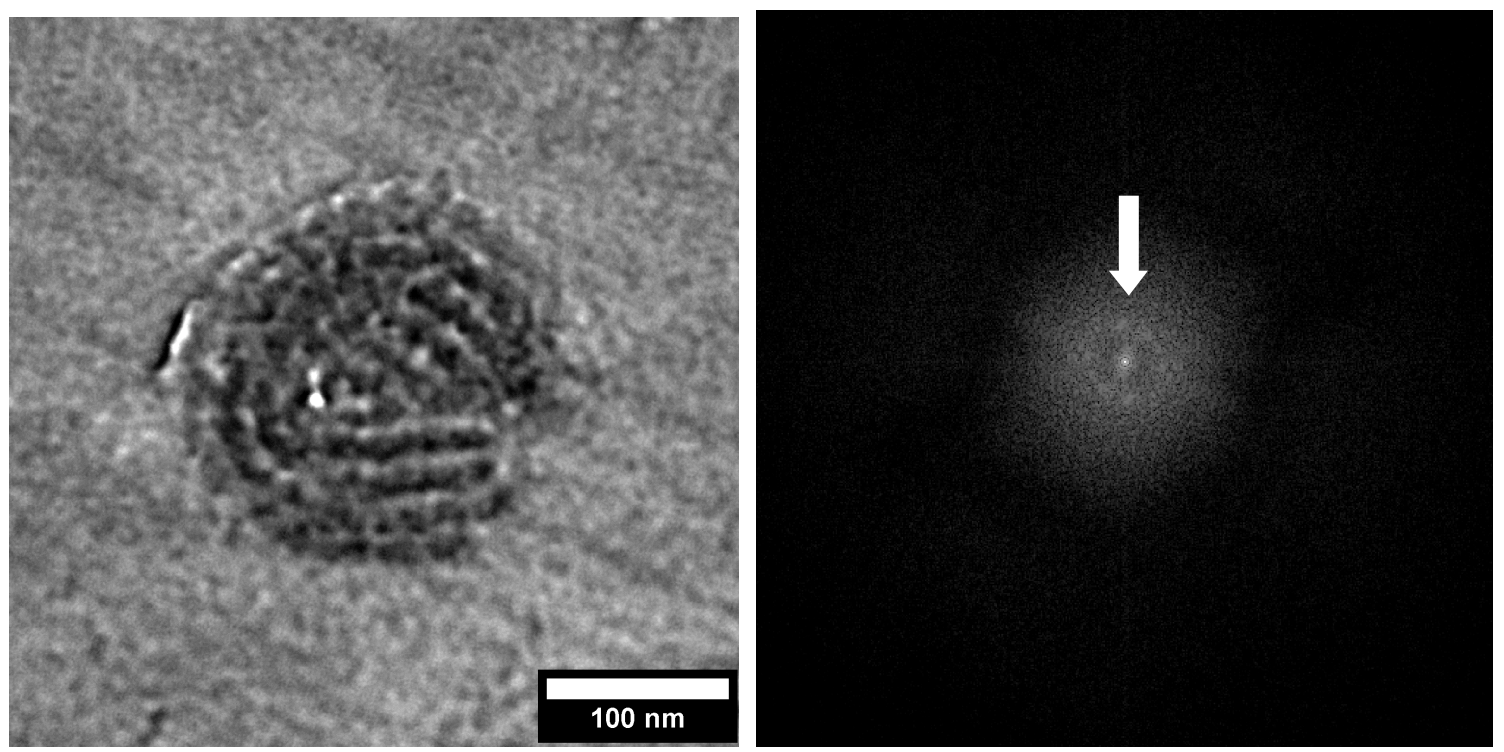

FIG. 1. Zero-loss filtered hole-free phase plate image of a melanin granule (left) and FFT (right) showing maxima corresponding to an $8 \mathrm{~nm}$ periodicity (marked by an arrow).
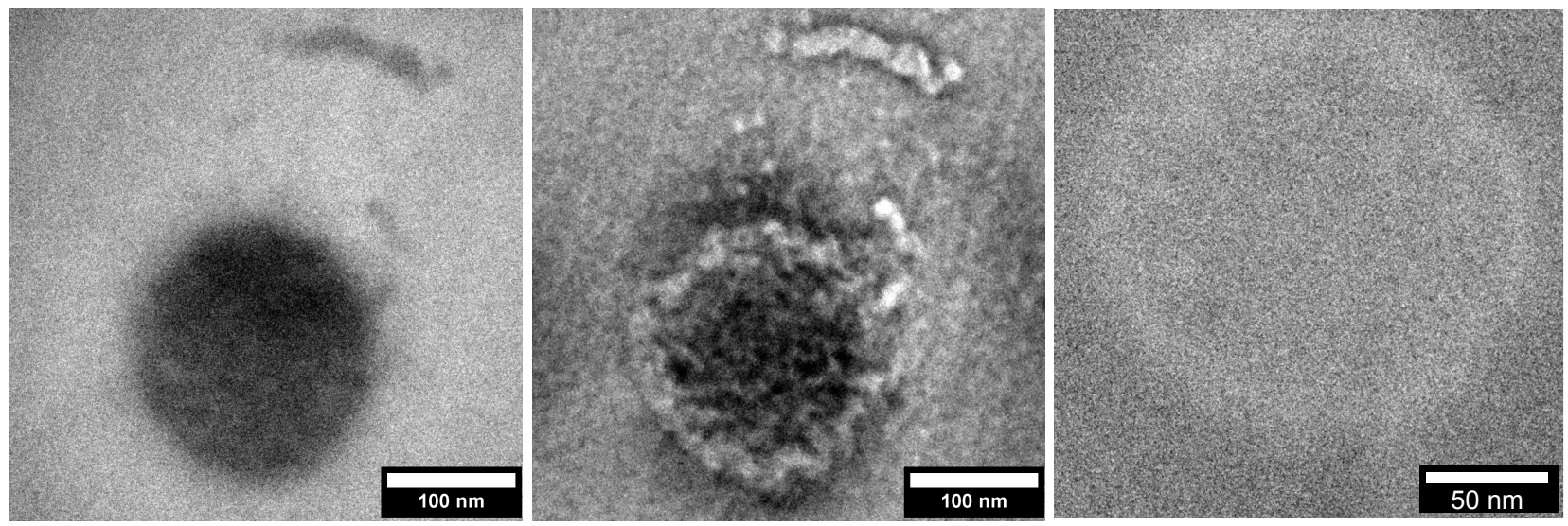

FIG. 2 Conventional TEM image of an unstained melanin granule (left). Melanin granule observed using a thin-film phase plate showing increased contrast core-shell structure (center). Energy filtered image obtained at $18 \mathrm{eV}$ with $5 \mathrm{eV}$ slit window (right) 